\title{
The splitting of solitary waves running over a shallower water
}

\author{
S.R. Pudjaprasetya ${ }^{\text {a, } *}$, E. Van Groesen ${ }^{\text {b }}$, E. Soewono ${ }^{\text {a }}$ \\ a Institut Teknologi Bandung, Department of Mathematics, Jl. Ganesha 10, Bandung 40132, Indonesia \\ b University of Twente, Department of Applied Mathematics, Enschede, The Netherlands
}

Received 23 December 1996; received in revised form 17 July 1998; accepted 3 August 1998

\begin{abstract}
The Korteweg-de Vries type of equation (called KdV-top) for uni-directional waves over a slowly varying bottom that has been derived by Van Groesen and Pudjaprasetya [E. van Groesen, S.R. Pudjaprasetya, Uni-directional waves over slowly varying bottom. Part I. Derivation of a KdV-type of equation, Wave Motion 18 (1993) 345-370.] is used to describe the splitting of solitary waves, running over shallower water, into two (or more) waves. Results of numerical computations with KdV-top are presented; qualitative and quantitative comparisons between the analytical and numerical results show a good agreement. (c) 1999 Elsevier Science B.V. All rights reserved.
\end{abstract}

\section{Introduction}

Consider a layer of ideal fluid (incompressible and irrotational) above topography $h(x)$. Let $\eta(x, t)$ be the wave height measured from an undisturbed water level. For rather long and rather low waves, amplitude $a=\mathcal{O}(\varepsilon)$, wavelength $l=\mathcal{O}(1 / \sqrt{\varepsilon})$, and for slow bottom variations $h^{\prime}(x)=\mathcal{O}\left(\epsilon^{\alpha}\right)$, with $\alpha>1$, a model equation for unidirectional waves was derived in [1]. The equation is a KdV-type of equation, which can be written as a Hamiltonian system

$$
\partial_{t} \eta=-\Gamma(x) \delta_{\eta} H(\eta)
$$

correct up to $\mathcal{O}\left(\varepsilon^{2}\right)$. The skew-symmetric operator $\Gamma(x)$ is given by

$$
\Gamma(x)=\frac{1}{2}\left(c(x) \partial_{x}+\partial_{x} c(x)\right),
$$

with $c(x)=\sqrt{g h(x)}$ and $g$ the gravitational acceleration. The Hamiltonian is

$$
H(\eta)=\int \frac{1}{2} \eta^{2}+\varepsilon\left(-\frac{1}{12} h^{2}(x) \eta_{x}^{2}+\frac{\eta^{3}}{4 h(x)}\right) \mathrm{d} x,
$$

\footnotetext{
*Corresponding author; e-mail: srpudjap@dns.math.itb.ac.id
} 
which is the total energy of the system. Eq. (1) is called the $K d V$-top equation. Being the Hamiltonian, the total energy $H(\eta)$ is conserved. Another conserved functional is

$$
C(\eta)=\int \frac{\eta}{\sqrt{c(x)}} \mathrm{d} x
$$

This functional is conserved since the kernel of the operator $\Gamma(x)$ is exactly $\delta C(\eta)=1 / \sqrt{c(x)}$. The momentum functional, defined as

$$
I(\eta)=\int \frac{\eta^{2}}{2 c(x)} \mathrm{d} x
$$

is not conserved, when $h$ is not constant.

When the bottom is flat $h(x)=h_{0}$, the KdV-top equation reduces to the standard KdV equation

$$
\partial_{t} \eta=-c_{0} \partial_{x} \delta_{\eta} \bar{H}\left(h_{0}, \eta\right)
$$

Note that the operator $\Gamma(x)$ reduces to $c_{0} \partial_{x}$, with $c_{0}=\sqrt{g h_{0}}$. The Hamiltonian $\bar{H}\left(h_{0}, \eta\right)$ is obtained from (3), by replacing $h$ with $h_{0}$. The KdV equation (6) is a completely integrable system that has infinitely many conserved functionals. The first two conserved functionals are mass and momentum, denoted by $\bar{C}\left(h_{0}, \eta\right)$ and $\bar{I}\left(h_{0}, \eta\right)$ which are the functionals given in (4) and (5), respectively, with $h, c$ replaced by $h_{0}, c_{0}$. Here we need one more conserved functional, and that is

$$
\bar{T}\left(h_{0}, \eta\right)=\int \eta^{4}-\frac{4}{3} h_{0}^{3} \eta \eta_{x}^{2}+\frac{4}{45} h_{0}^{6} \eta_{x x}^{2} \mathrm{~d} x .
$$

Our aim is to study solitary wave deformations due to decreasing depth using the KdV-top equation (1). Previously, the deformation of solitary waves due to a slowly decreasing depth has been studied in [2]. In that paper, the deformation is approximated by a quasi-homogeneous (Q-H) succession of solitary waves plus a flat tail behind the wave. While running up, the growing solitary waves could not take all the mass accumulated by decreasing depth; and so this flat tail is supplemented to the solitary wave in order to maintain mass conservation.

In this paper, we restrict ourselves to a topography which is a smooth transition between two constant depths, decreasing in the direction of wave propagation. A detail description of this problem can be found in the thesis by Pudjaprasetya [3]. When the wave arrives at the region of a shallower constant depth, there will be no more mass surplus, and since the tail travels slower than the main wave, the tail will split from the wave. Numerical computations solving the KdV-top by Van Beckum [4] confirm this; further, it shows that above a shallower constant depth the tail starts to deform like a solitary wave.

Motivated by that numerical result, which might be considered as the 'exact' description of the phenomenon, here we describe the splitting process of a solitary wave as a quasi-homogeneous (Q-H) succession of two-soliton shapes. The change of parameters determining the two-soliton shape is obtained from conservation of energy and mass.

We will also show that the splitting of a solitary wave is also evident from the fact that the manifold of two-soliton shapes can be viewed as an unfolding of the one-solitons manifold: starting with a one-soliton, bottom variations will cause the values of the parameters to change, and to enter the region of two-solitons.

The Q-H two-soliton approximation and the numerical computation show qualitatively the same deformation of solitary wave running up: amplitude is increasing, wavelength and velocity are decreasing and the wave splits into two waves of solitary wave shape.

We also compare the splitting described here with the results of inverse scattering (IS) theory. The difference between the Q-H approximation and the IS approach by Johnson [5] is that the Q-H approximation gives the evolution of a solitary wave during run up, in which is not the case in the IS approach. However, when the two-soliton are already separated, the amplitudes, momenta and velocities of the two single-solitons obtained from both approaches show a good agreement. 
Referring to those numerical results, we discuss the validity of the Q-H two-soliton approximation and give suggestions for further approximations. We also discuss how this approximation relates to the Q-H approximation based on solitary waves with tail described in [2].

The organization of this paper is as follows. In Section 2 we describe the unfolding of the manifold of two-solitons from the manifold of one-solitons in the flat bottom case. The results of numerical computations for KdV-top are presented in Section 3. In Section 4 we study the solitary wave deformation using the Q-H approximation based on two-soliton shapes. In Section 5, a qualitative comparison between the Q-H approximation and numerical calculations is given. Also, the Q-H approximation, the IS approach and numerical computations are compared quantitatively. In Section 6 we discuss the validity of the Q-H two-soliton approximation.

\section{Unfolding of one-soliton to two-soliton for KdV flat bottom}

The $\mathrm{KdV}$ equation (6) has solitary wave solutions and $N$-soliton solutions, with $N \geq 2$. A solitary wave shape can be characterized as the extremizer of the energy $\bar{H}\left(h_{0}, \eta\right)$ on level set of momentum $\bar{I}\left(h_{0}, \eta\right)=\gamma$ :

$$
\mathcal{S}\left(h_{0}, \gamma\right) \in \operatorname{Max}_{\eta}\left\{\bar{H}\left(h_{0}, \eta\right) \mid \bar{I}\left(h_{0}, \eta\right)=\gamma, \eta \in \mathcal{D}\right\}
$$

with $\mathcal{D}$ the set of functions that decay sufficiently fast at infinity, together with their derivatives. The solitary wave shape with the crest-position at $\varphi$ is given by

$$
\mathcal{S}^{(1)}\left(h_{0}, \gamma\right)(x-\varphi)=a \operatorname{sech}^{2} b(x-\varphi)
$$

with $a$ and $b$ functions of $h_{0}$ and $\gamma$. A solitary wave solution of the $\mathrm{KdV}$ equation is then $\mathcal{S}^{(1)}\left(h_{0}, \gamma\right)(x-\lambda t)$, with $\lambda$ a function of $h_{0}$ and $\gamma$.

A two-soliton shape of the KdV equation can be characterized analogously to the solitary wave shape in (8), but with an additional constraint:

$$
\tilde{\mathcal{S}}^{(2)}\left(h_{0}, \gamma, \tau, \varphi_{-}, \varphi_{+}\right) \in \operatorname{Max}_{\eta}\left\{\bar{H}\left(h_{0}, \eta\right) \mid \bar{I}\left(h_{0}, \eta\right)=\gamma, \bar{T}\left(h_{0}, \eta\right)=\tau, \eta \in \mathcal{D}\right\}
$$

Here, $\bar{T}\left(h_{0}, \eta\right)$ is given by (7). As $t \rightarrow \pm \infty$ the two-soliton solution behaves, like two single-solitons far apart from each other. When the two-soliton are separated, parameters $\varphi_{-}$and $\varphi_{+}$denote the crest-positions of the small and the high solitary wave respectively. Suppose $\mathcal{S}^{(1)}\left(h_{0}, \gamma_{-}\right)$and $\mathcal{S}^{(1)}\left(h_{0}, \gamma_{+}\right)$are the two single-solitons of momentum $\gamma_{-}, \gamma_{+}$respectively, that are the constituent waves of the two-soliton. Since the two functionals $\bar{T}\left(h_{0}, \eta\right)$ and $\bar{I}\left(h_{0}, \eta\right)$ are conserved, their values evaluated at the two-soliton $\mathcal{S}^{(2)}$ equal the sum of their values evaluated at $\mathcal{S}^{(1)}\left(h_{0}, \gamma_{-}\right)$ and at $\mathcal{S}^{(1)}\left(h_{0}, \gamma_{+}\right)$. Hence we have a relation between $\gamma, \tau$ and $\gamma_{-}, \gamma_{+}$as follows

$$
\begin{aligned}
& \gamma=\gamma_{-}+\gamma_{+}, \\
& \tau=\mathcal{T}\left(h_{0}, \gamma_{-}\right)+\mathcal{T}\left(h_{0}, \gamma_{+}\right),
\end{aligned}
$$

where $\left.\mathcal{T}\left(h_{0}, \gamma_{ \pm}\right) \equiv \bar{T}\left(h_{0}, \eta\right)\right|_{\mathcal{S}^{(1)}\left(h_{0}, \gamma_{ \pm}\right)}$; for a fixed $h_{0}, \mathcal{T}$ is a convex function of $\gamma$. To interpret (10) and (11), we introduce $\xi=\gamma_{-}$and consider the right-hand side of (11) as a function of $\xi$ :

$$
f(\xi): e \mathcal{T}\left(h_{0}, \xi\right)+\mathcal{T}\left(h_{0}, \gamma-\xi\right), \quad \text { with } \quad \xi \in[0, \gamma / 2]
$$

The function $f(\xi)$ is a convex function of $\gamma$ (since $\mathcal{T}$ is convex), see Fig. 1. Moreover, $f(0)=f(\gamma)$ and $f$ is symmetric around $\gamma / 2$. Hence, for given values of $\gamma$ and $\tau$ we obtain $\gamma_{+}$and $\gamma_{-}$as the points of intersection of the horizontal line $\tau$ with the curve $f(\xi)$. If we denote the minimum of $f(\xi)$ by $\underline{\tau}\left(h_{0} ; \gamma\right) \equiv f(\gamma / 2)=$ $(1 / 2)^{4 / 3} \mathcal{T}\left(h_{0}, \gamma\right)$, we conclude that for each $\tau \in\left(\underline{\tau}\left(h_{0}, \gamma\right), \mathcal{T}\left(h_{0}, \gamma\right)\right)$ there exists a unique pair $\left(\gamma_{-}, \gamma_{+}\right)$, with 


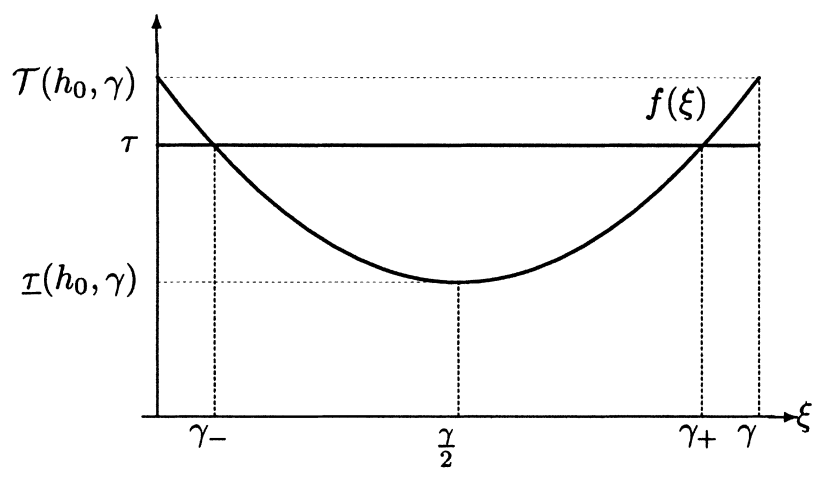

Fig. 1. The convex function $f$ symmetric around $\gamma / 2$.

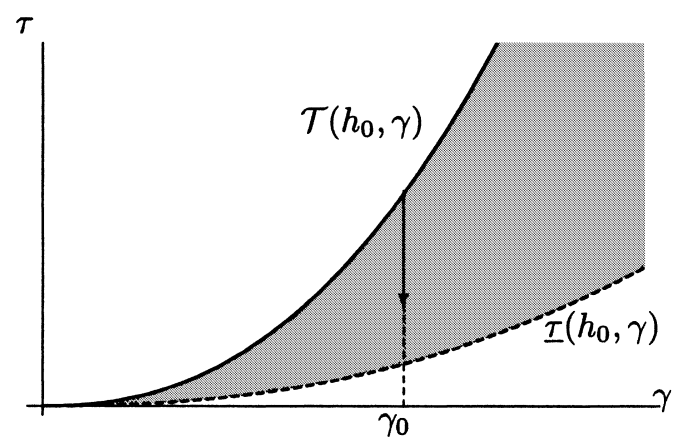

Fig. 2. The one-soliton curve $\mathcal{T}\left(h_{0}, \gamma\right)$ and below it the two-soliton region (shaded).

$0<\gamma_{-}<\gamma_{+}<\gamma$, that solves the relations (10) and (11). Therefore, we can replace the parameters $\gamma, \tau$ with $\gamma_{-}, \gamma_{+}$, and we denote the two-soliton shape as

$$
\tilde{\mathcal{S}}^{(2)}\left(h_{0}, \gamma, \tau, \varphi_{-}, \varphi_{+}\right) \equiv \mathcal{S}^{(2)}\left(h_{0}, \gamma_{-}, \gamma_{+}, \varphi_{-}, \varphi_{+}\right) .
$$

We conclude that the manifold of solitary wave shapes and two-soliton shapes are given by

$$
\begin{aligned}
& \mathcal{M}_{1}=\left\{\mathcal{S}\left(h_{0}, \gamma\right)(x-\varphi) \mid \gamma, \varphi \in \mathbb{R}\right\}, \\
& \mathcal{M}_{2}=\left\{\mathcal{S}^{(2)}\left(h_{0}, \gamma_{-}, \gamma_{+}, \varphi_{+}, \varphi_{-}\right) \mid \gamma_{-}, \gamma_{+}, \varphi_{+}, \varphi_{-} \in \mathbb{R}\right\} .
\end{aligned}
$$

Consider the maximization problem (9) on level set $\bar{T}\left(h_{0}, \eta\right)=\mathcal{T}\left(h_{0}, \gamma\right)$, for any $\gamma \in \mathbb{R}$. Clearly, it yields the one-soliton $\mathcal{S}\left(h_{0}, \gamma\right)$. Other level sets of $\bar{T}\left(h_{0}, \eta\right)$ in (9) that yield two-soliton shapes define a region in $\gamma, \tau$-plane, illustrated in Fig. 2. Hence, $\mathcal{M}_{2}$ is an unfolding of $\mathcal{M}_{1}$. In this flat bottom case, for a fixed $\gamma_{0}$ we can 'switch' from a one-soliton to a two-soliton simply by varying the value of $\tau$.

\section{Numerical simulations of soliton splitting}

In this section we present numerical simulations of solitary waves during run up. In the numerical frame work, we deal with a water tank of finite length, therefore as the initial wave we take a solitary wave which is composed from a cnoidal wave; when the trough of cnoidal wave is very long and the wave is confined to a relatively small region, the cnoidal wave resembles a solitary wave shape. 
For a fixed wavelength $l$, the cnoidal wave shape with momentum $\hat{\gamma}$, above a flat bottom at depth $h_{0}$, denoted as $C n\left(c_{0}, \hat{\gamma}\right)$, can be characterized as a solution of the constrained maximization problem:

$$
C n\left(h_{0}, \hat{\gamma}\right) \in \operatorname{Max}_{\eta}\left\{\bar{H}\left(h_{0}, \eta\right) \mid \bar{I}\left(h_{0}, \eta\right)=\hat{\gamma}, \bar{C}\left(h_{0}, \eta\right)=0, \eta \in C_{\mathrm{per}}^{\infty}\right\}
$$

with $C_{\text {per }}^{\infty}$ the functions set consists of infinitely differentiable periodic functions, with period $l$. The conserved functionals of $\mathrm{KdV}$ equation (6), i.e. energy $\bar{H}\left(h_{0}, \eta\right)$, mass $\bar{C}\left(h_{0}, \eta\right)$ and momentum $\bar{I}\left(h_{0}, \eta\right)$, are integrals over the interval $[-l / 2, l / 2]$. The constraint $\bar{C}(\eta)=0$ means that the cnoidal wave is constructed to have zero mass. In finding the critical point of (15) we use the steepest descent method; a complete explanation of the algorithm can be found in [4].

We then let this wave evolve according to the discretized $\mathrm{KdV}$-top equation. The $\mathrm{KdV}$-top equation is discretized using a direct Fourier truncation method. Let $\hat{\eta}(t)$ denote the vector consisting of coefficients of a Fourier truncation of $\eta(x, t)$. The evolution of $\hat{\eta}(t)$ is described by a Hamiltonian equation:

$$
\frac{\mathrm{d} \hat{\eta}}{\mathrm{d} t}=\hat{\Gamma} \nabla \hat{H}(\hat{\eta})
$$

where $\nabla \hat{H}$ is obtained from the Hamiltonian (3), after substituting the Fourier truncation of $\eta$, and taking the partial derivatives with respect to elements of $\hat{\eta}$. The operator $\hat{\Gamma}$ is a square matrix, acting on the vector $\nabla \hat{H}$. Observe that the inhomogeneity from the topography, i.e. $c(x)$, is treated in a special way in doing this. Eq. (16) is integrated using the Runge-Kutta method. For the complete description, see [4].

The numerical programme solving the KdV-top equation, used in this paper and also the programme constructing a cnoidal wave from (15), are taken from the software package WAVEPACK, composed by Van Beckum and Djohan [6].

We choose a topography which is a smooth transition between two constant depths, decreasing linearly in the direction of wave propagation from $h_{0}$ to $h_{1}$. In these numerical simulations we expect that above the shallower constant depth, the wave will split into two-soliton. In order to get simulations where the two single-solitons are really separated, we need to have a numerical wave tank which is quite long, which means a long wavelength. Increasing the wavelength for a steep cnoidal wave requires more Fourier modes. Since the WAVEPACK code can use 64 Fourier modes at most, this limits the longest wavelength to a length of $l=70 \mathrm{~m}$, which is taken in the following computation.

For all computations we use an initial solitary wave that is constructed from a cnoidal wave of amplitude $0.1 \mathrm{~m}$, above a layer of water with a constant depth $h_{0}=0.5 \mathrm{~m}$; the cnoidal wave is lifted so that its troughs are on the zero water level. This lifted cnoidal wave, in the limiting case $(l \rightarrow \infty)$, is a solitary wave of amplitude $0.1 \mathrm{~m}$, above a constant depth $h_{0}=0.5 \mathrm{~m}$, see Lemma 4.1 in [3]; this lifted cnoidal wave is a good approximation of a solitary wave that travels undisturbed in shape above a flat bottom $h_{0}=0.5 \mathrm{~m}$.

The calculated waves are shown at subsequent stages of evolution $t=0,4, \ldots, 28$ in Fig. 3 (top), (middle) and (bottom), using three choices of the constant depths $h_{1}=0.3 \mathrm{~m}, 0.35 \mathrm{~m}$ and $0.4 \mathrm{~m}$, respectively. In the evolution equation we take $\varepsilon=0.7$. (In [3] we show that the result of the KdV-top model for $\varepsilon=0.7$ matches best with those of the full governing equations, over a long range of wave amplitudes).

Referring to Fig. 3, the effect of decreasing depth on the wave is four-fold: the wave becomes higher and steeper, its speed decreases and it splits into two waves with a shape that closely resembles that of a solitary wave. Besides the two-soliton, we also observe an oscillating tail behind the small wave. A closer look at Fig. 3 (middle) and (bottom), shows that the second soliton and the oscillating tail are not really separated, and that they form a dip directly behind the second soliton. This dip is not clearly observable in Fig. 3 (top). We will explain this in Section 6.

\section{Quasi-homogeneous two-soliton approximation}

In this section we study the deformation of a solitary wave, say of momentum $\gamma_{0}$, which is initially above a flat bottom at depth $h_{0}$, denoted by $\mathcal{S}_{0}\left(h_{0}, \gamma_{0}\right)$, running up to a shallower constant depth. We use the Q-H approximation 

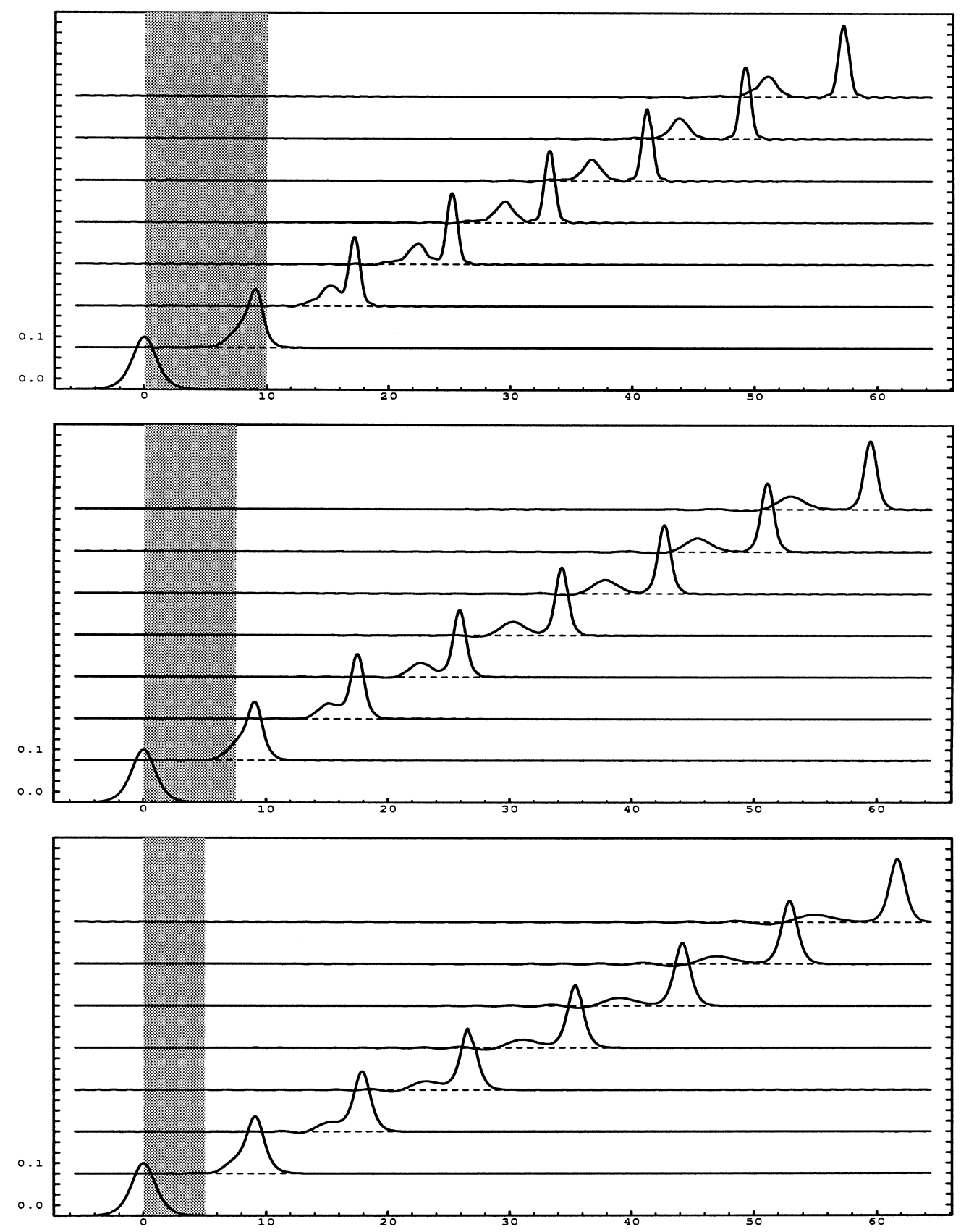

Fig. 3. Numerical simulations of soliton splitting due to a decreasing bottom with depths $0.3 \mathrm{~m}$ (top), $0.35 \mathrm{~m}$ (middle), $0.4 \mathrm{~m}$ (bottom).

based on two soliton shapes to describe the solitary wave deformation as a gradually developing separation process of a two-soliton.

The idea of the Q-H approximation with two-soliton shapes is as follows. Suppose that at a certain time the wave is at 'position' $x=\varphi$; for given $\gamma$ and $\tau$, its shape is approximated by a two-soliton shape above a constant bottom at depth $h(\varphi)$, denoted as $\tilde{\mathcal{S}}^{(2)}\left(h \equiv h(\varphi), \gamma, \tau, \varphi_{-}, \varphi_{+}\right)$, see Fig. 4. From now on the notation $h$ is used to denote 


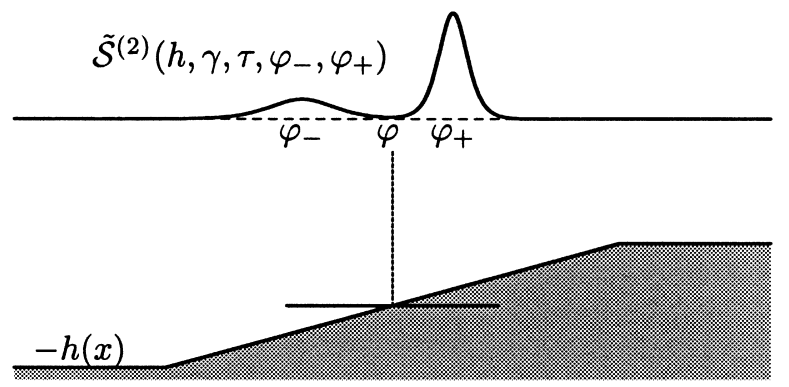

Fig. 4. The two-soliton shape $\tilde{\mathcal{S}}^{(2)}\left(h, \gamma, \tau, \varphi_{-}, \varphi_{+}\right)$above a flat bottom $h(x)=h(\varphi)$ approximates the wave shape at $x=\varphi$.

$h(\varphi)$. The two-soliton shape $\tilde{\mathcal{S}}^{(2)}\left(h, \gamma, \tau, \varphi_{-}, \varphi_{+}\right)$is characterized by the constrained maximization problem like (9) with $h_{0}$ replaced by $h$.

We define the position $\varphi$ to be the 'center of gravity' of the wave, using the (generalized) mass density, as follows:

$$
\varphi=\frac{\int(x \eta / \sqrt{c(x)}) \mathrm{d} x}{\int(\eta / \sqrt{c(x)}) \mathrm{d} x} .
$$

Note that in (17) we use the Casimir as the mass-like functional, which is a constant of the motion in order to makes the later calculations simple.

In Section 2 we showed that the parameters $\gamma, \tau$ can be replaced by $\gamma_{-}, \gamma_{+}$, and the two-soliton shape is denoted as $\mathcal{S}^{(2)}\left(h, \gamma_{-}, \gamma_{+}, \varphi_{-}, \varphi_{+}\right)$. Then the Q-H two-soliton approximation is

$$
t \mapsto \mathcal{S}^{(2)}\left(h(\varphi(t)), \gamma_{-}(t), \gamma_{+}(t), \varphi_{-}(t), \varphi_{+}(t)\right),
$$

where the evolution for $\varphi(t), \gamma_{-}(t)$ and $\gamma_{+}(t)$ has to be determined. The parameters $\varphi_{-}(t)$ and $\varphi_{+}(t)$ will be determined through the velocity of each single-soliton.

\subsection{Parameter dynamics from energy and mass conservation}

For exact solutions of the $\mathrm{KdV}$-top equation, the energy $H(\eta)$ and mass $C(\eta)$ are conserved, therefore we invoke energy and mass conservation for our Q-H approximation. Let $E_{0}$ and $M_{0}$ be the initial energy and mass evaluated at the initial solitary wave $\mathcal{S}_{0}\left(h_{0}, \gamma_{0}\right)$, so $E_{0} \equiv \bar{H}\left(h_{0}, \mathcal{S}_{0}\right)$ and $M_{0} \equiv \bar{C}\left(h_{0}, \mathcal{S}_{0}\right)$. Invoking energy and mass conservation for the Q-H two-soliton approximation (18) yields

$$
\bar{H}\left(h, \mathcal{S}^{(2)}\right)=E_{0}, \quad \bar{C}\left(h, \mathcal{S}^{(2)}\right)=M_{0} .
$$

Eq. (19) have a clear geometrical interpretation, meaning that the evolution takes place in the intersection of the level sets of energy and mass. In principal, Eq. (19) determine the dynamics of our Q-H approximation, i.e. $\gamma-$ and $\gamma_{+}$as functions of $h$.

Recall that as $t \rightarrow \pm \infty$, a two-soliton solution has the form of two solitary waves $\mathcal{S}\left(h, \gamma_{-}\right)$and $\mathcal{S}\left(h, \gamma_{+}\right)$, separated far apart from each other. Since the functionals $\bar{H}(h, \eta)$ and $\bar{C}(h, \eta)$ are conserved functionals of the $\mathrm{KdV}$ equation, so their values evaluated at the two-soliton equal the sum of their values evaluated at $\mathcal{S}\left(h, \gamma_{-}\right)$and at $\mathcal{S}\left(h, \gamma_{+}\right)$. Hence, the energy and mass conservation read

$$
\mathcal{H}\left(h, \gamma_{-}\right)+\mathcal{H}\left(h, \gamma_{+}\right)=E_{0}, \quad \mathcal{C}\left(h, \gamma_{-}\right)+\mathcal{C}\left(h, \gamma_{+}\right)=M_{0}
$$

where $\mathcal{H}\left(h, \gamma_{ \pm}\right) \equiv \bar{H}\left(h, \mathcal{S}\left(h, \gamma_{ \pm}\right)\right)$and $\mathcal{C}\left(h, \gamma_{ \pm}\right) \equiv \bar{C}\left(h, \mathcal{S}\left(h, \gamma_{ \pm}\right)\right)$denote the energy and mass respectively of each solitary wave $\mathcal{S}\left(h, \gamma_{-}\right)$and $\mathcal{S}\left(h, \gamma_{+}\right)$. A simple, but interesting, geometrical interpretation of (20) can be found 


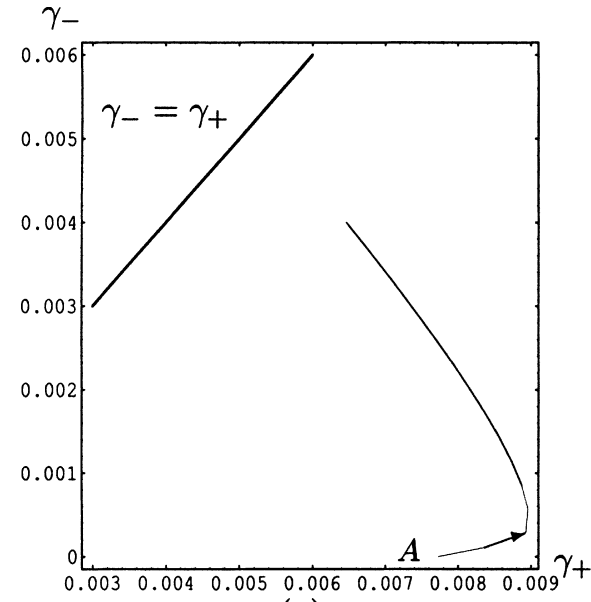

(a)

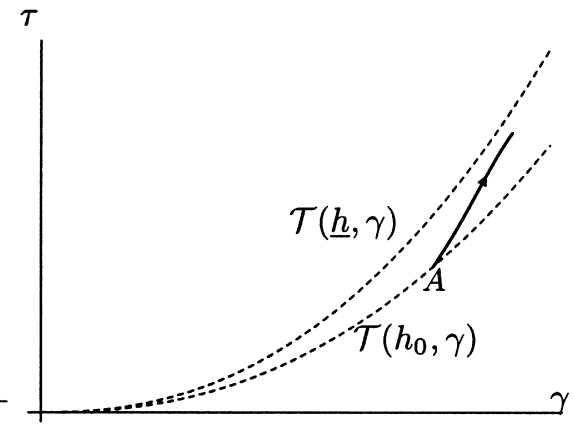

(b)

Fig. 5. Trajectories of a running up soliton in two parameter-planes of interest. The arrows show the directions for a decreasing depth. Point A represents the initial solitary wave $\mathcal{S}_{0}\left(h_{0}, \gamma_{0}\right)$.

in [7]. Explicit solutions can be written down when the $\varepsilon$-term in the energy is neglected, and they read

$$
\gamma_{ \pm}(h)=\frac{1}{8}\left(\frac{h_{0}}{h}\right)^{11 / 4} \gamma_{0}\left\{1 \pm\left(\frac{h_{0}}{h}\right)^{-2 / 3} \sqrt{\frac{4}{3}-\frac{1}{3}\left(\frac{h_{0}}{h}\right)^{9 / 4}}\right\}^{3}
$$

which are valid for $h \in\left[(1 / 4)^{4 / 9} h_{0}, h_{0}\right]$.

It turns out that solutions (21) are a good approximations on the interval of interest, therefore, later on we will proceed with (21).

From (21) it is clear that the momenta $\gamma_{-}$and $\gamma_{+}$are directly related to the depth ratio. For a depth ratio that is close to one, i.e. $h_{0} / h \approx 1$, the term inside the square root in (21) is close to, but less than one; therefore, $\gamma_{-}$is very small which means that initially the second soliton is very small. To be more precise, for $h<h_{0}$, but close to $h_{0}$, one can show that $\gamma_{-} \approx K\left(\gamma_{+}-\gamma_{0}\right)^{3}$, for some constant $K$.

Fig. 5 shows the evolution of a soliton running up, given by (21), in two sets of parameters, with $h$ as the parameter along the curves:

$$
\begin{aligned}
& \left\{\left(\gamma_{-}(h), \gamma_{+}(h)\right) \mid h_{1} \leq h \leq h_{0}\right\}, \\
& \left\{\left(\gamma, \mathcal{T}\left(h, \gamma_{-}\right)+\mathcal{T}\left(h, \gamma_{+}\right)\right) \mid h_{1} \leq h \leq h_{0}\right\} .
\end{aligned}
$$

Here $h_{1}=(1 / 4)^{4 / 9} h_{0}$ is the limiting value at which $\gamma_{-}$becomes equal to $\gamma_{+}$; the (ir)relevance of this limit will be discussed in Section 6. Recall that $\mathcal{T}(h, \gamma)$ is the value of $\bar{T}(h, \eta)$ evaluated at a single-soliton; then $\mathcal{T}\left(h, \gamma_{-}\right)+\mathcal{T}\left(h, \gamma_{+}\right)$is the actual value of $\bar{T}(h, \eta)$ evaluated at the two-soliton $\mathcal{S}^{(2)}$.

Consider Fig. 5(b); recall that for each fixed $h$, the curve $\gamma \mapsto \mathcal{T}(h, \gamma)$ is the one-soliton curve; below this curve we have a region of two-solitons. The trajectory (23) starts at the initial solitary wave $\mathcal{S}_{0}$ which is represented on the curve $\mathcal{T}\left(h_{0}, \gamma\right)$ by $A$; then decreasing the parameter $h$ to some value $\underline{h}<h_{0}$, the final point lies below the curve $\mathcal{T}(\underline{h}, \gamma)$, that it represents a two-soliton. In Section 5 we compare the momentum parameters given by (21) with those obtained from the IS method.

Let us recapitulate what we already obtained: when the wave has a position $x=\varphi$, we approximate the wave profile with the two-soliton shape $\mathcal{S}^{(2)}\left(h, \gamma_{-}(h), \gamma_{+}(h), \varphi_{-}, \varphi_{+}\right)$with $\gamma_{-}(h), \gamma_{+}(h)$ given by (21) and $h=h(\varphi)$. The values of the parameters $\varphi_{-}, \varphi_{+}$follow from $\dot{\varphi}_{ \pm} \equiv \lambda_{ \pm}$, the velocities of two single solitons when separated. 
We still have to find the dynamics of $\varphi(t)$. This is obtained by differentiating (17) with respect to $t$; for an exact solution of KdV-top we have the following dynamics:

$$
C(\eta) \dot{\varphi}=-\int x \frac{\Gamma(x) \delta H}{\sqrt{c(x)}} \mathrm{d} x,=-\int x \partial_{x}(\sqrt{c(x)} \delta H) \mathrm{d} x,=\int \sqrt{c(x)} \delta H \mathrm{~d} x .
$$

Substituting $\eta=\mathcal{S}^{(2)}\left(h, \gamma_{-}, \gamma_{+}, \varphi_{-}, \varphi_{+}\right)$into (24), the right-hand side can be calculated leading to

$$
\lambda_{-} \mathcal{C}\left(h, \gamma_{-}\right)+\lambda_{+} \mathcal{C}\left(h, \gamma_{+}\right)+\mathcal{O}\left(\varepsilon^{2}\right) .
$$

In this derivation, we approximated $\mathcal{S}^{(2)} \approx \mathcal{S}^{(1)}\left(h, \gamma_{-}\right)+\mathcal{S}^{(1)}\left(h, \gamma_{+}\right)$, which is valid if the two single-solitons are already separated. Moreover, since the Casimir $C(\eta)$ is a constant of the motion, its value evaluated at $\mathcal{S}^{(2)}$ equal the sum of its value evaluated at $\mathcal{S}^{(1)}\left(h, \gamma_{-}\right)$and at $\mathcal{S}^{(1)}\left(h, \gamma_{+}\right)$. Hence, the left-hand side of (24) reads

$$
C\left(\mathcal{S}^{(2)}\right) \dot{\varphi}=\left(\mathcal{C}\left(h, \gamma_{-}\right)+\mathcal{C}\left(h, \gamma_{+}\right)\right) \dot{\varphi} .
$$

Taken together, we find that the dynamics for the center of gravity is a weighted average of the velocities of the two constituent waves, with the fractions of the mass of the waves as weights:

$$
\dot{\varphi}=\lambda_{-} \frac{\mathcal{C}\left(h, \gamma_{-}\right)}{\mathcal{C}\left(h, \gamma_{-}\right)+\mathcal{C}\left(h, \gamma_{+}\right)}+\lambda_{+} \frac{\mathcal{C}\left(h, \gamma_{+}\right)}{\mathcal{C}\left(h, \gamma_{-}\right)+\mathcal{C}\left(h, \gamma_{+}\right)},
$$

which correct up to $\mathcal{O}\left(\varepsilon^{2}\right)$.

Finally, we present some simulations of solitary wave evolutions during run up, obtained from this Q-H method. For any fixed time $t$, the 'position' $x=\varphi(t)$ is determined by (26), then the momentum parameters that characterize the two-solitons are $\gamma_{ \pm}(h(\varphi))$, obtained from (21), and the wave profile at that time is a two-soliton shape $\mathcal{S}^{(2)}\left(h, \gamma_{-}(h), \gamma_{+}(h), \varphi_{-}, \varphi_{+}\right)$.

Here we use the configuration that was used for numerical computations in Section 3: an initial solitary wave of amplitude $0.1 \mathrm{~m}$ above a constant depth $h_{0}=0.5 \mathrm{~m}$; the topography is a smooth transition between two constant depths, decreasing linearly in the direction of wave propagation from $h_{0}$ to three values of $h_{1}=0.3 \mathrm{~m}, 0.35 \mathrm{~m}$ and $0.4 \mathrm{~m}$. In Fig. 6 we plot the wave profiles at the subsequent stages of the evolution $t=0,4, \ldots, 28$. The regions of decreasing depth are indicated in grey. The positions of the two wave-crests are represented by the thin lines; their gradients represent the velocities of the two waves $\lambda_{ \pm}$, and the gradient of the other line represents the linear velocity $c_{1}=\sqrt{g h_{1}}$. For the same reason as in Section 3, for these simulations we take $\varepsilon=0.7$. In Section 5 we compare these analytical simulations with those obtained numerically.

\section{Comparison of analytical, numerical and inverse scattering results}

For a qualitative comparison between the analytical and numerical results, consider Figs. 6 and 3. Apart from the oscillating tail, that is missing in the Q-H two-soliton approximation, the analytical description agrees qualitatively very well with the numerical simulations.

For a quantitative comparison of observable quantities, such as amplitudes, momenta and velocities of the two single-solitons, we will include the result of the IS approach. Therefore, here we describe briefly the IS approach by Johnson [5].

Johnson [5] (see also [8]) approaches the same problem in a different way. Basically his approach consists of two steps. Firstly, during run up from $h_{0}$ to $h_{1}$ the wave is scaled in a simple way: from $\eta_{0}$ to $\left(h_{0} / h_{1}\right)^{1 / 4} \eta_{0}$. This 'evolution' is not justified by any equation, nor does it take into account details of the changing topography between the two constant depths. Nevertheless, this scaling evolution does satisfy the property that mass and energy are conserved during this transition. Secondly, the distortion of $\left(h_{0} / h_{1}\right)^{1 / 4} \eta_{0}$ above the constant depth $h_{1}$ is obtained 

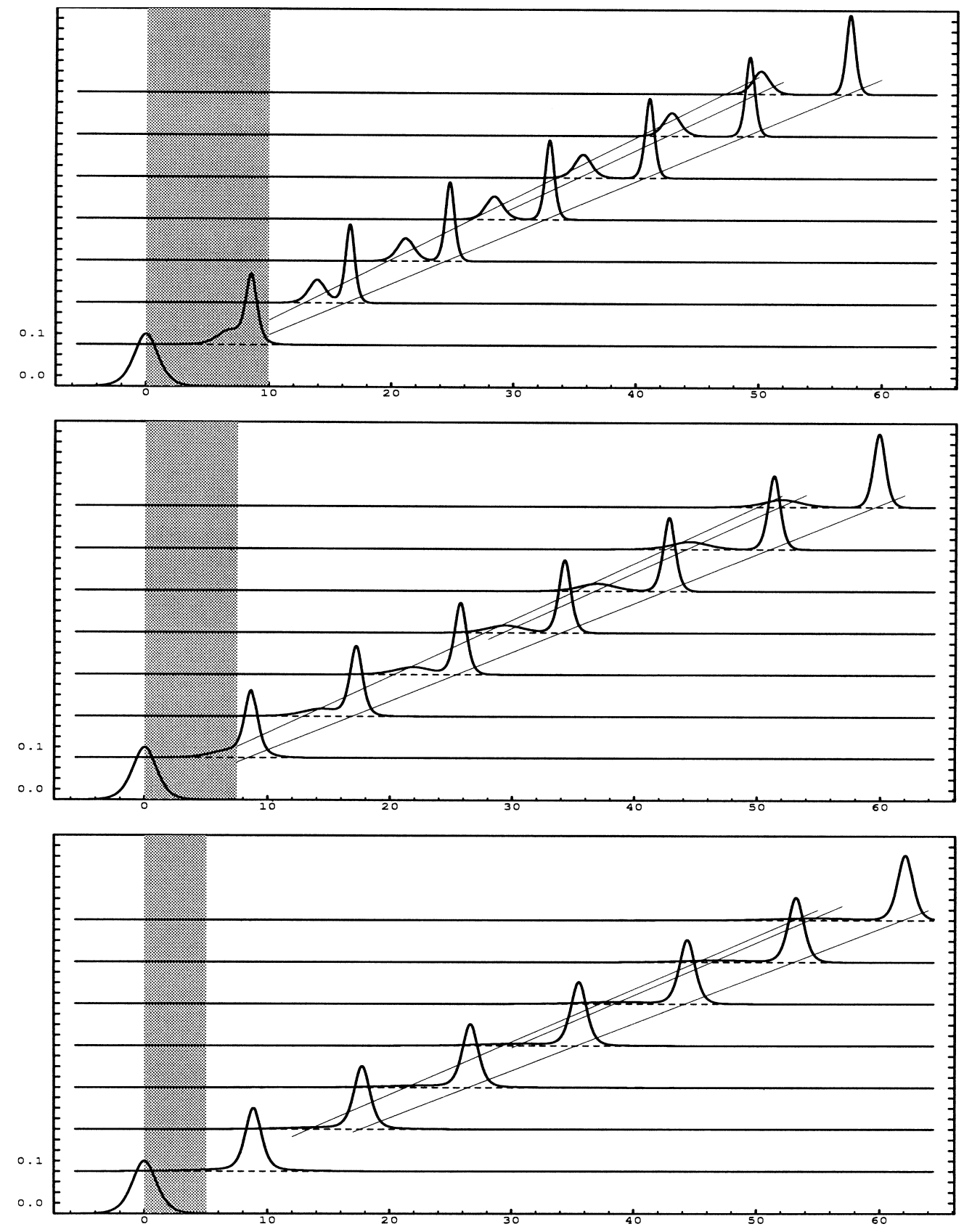

Fig. 6. Analytical approximations of soliton splitting due to decreasing bottom with depths $h_{1}=0.3 \mathrm{~m}$ (top), $h_{1}=0.35 \mathrm{~m}$ (middle), $h_{1}=0.4 \mathrm{~m}$ (bottom).

by applying the IS method to the standard $\mathrm{KdV}$ equation. As a consequence, conservation of mass and energy holds for the whole evolution. Introducing discrete values (eigendepths) as

$$
\hbar_{N} \equiv\left(\frac{2}{N(N+1)}\right)^{4 / 9} h_{0}, \quad \text { for } \quad N \geq 1
$$



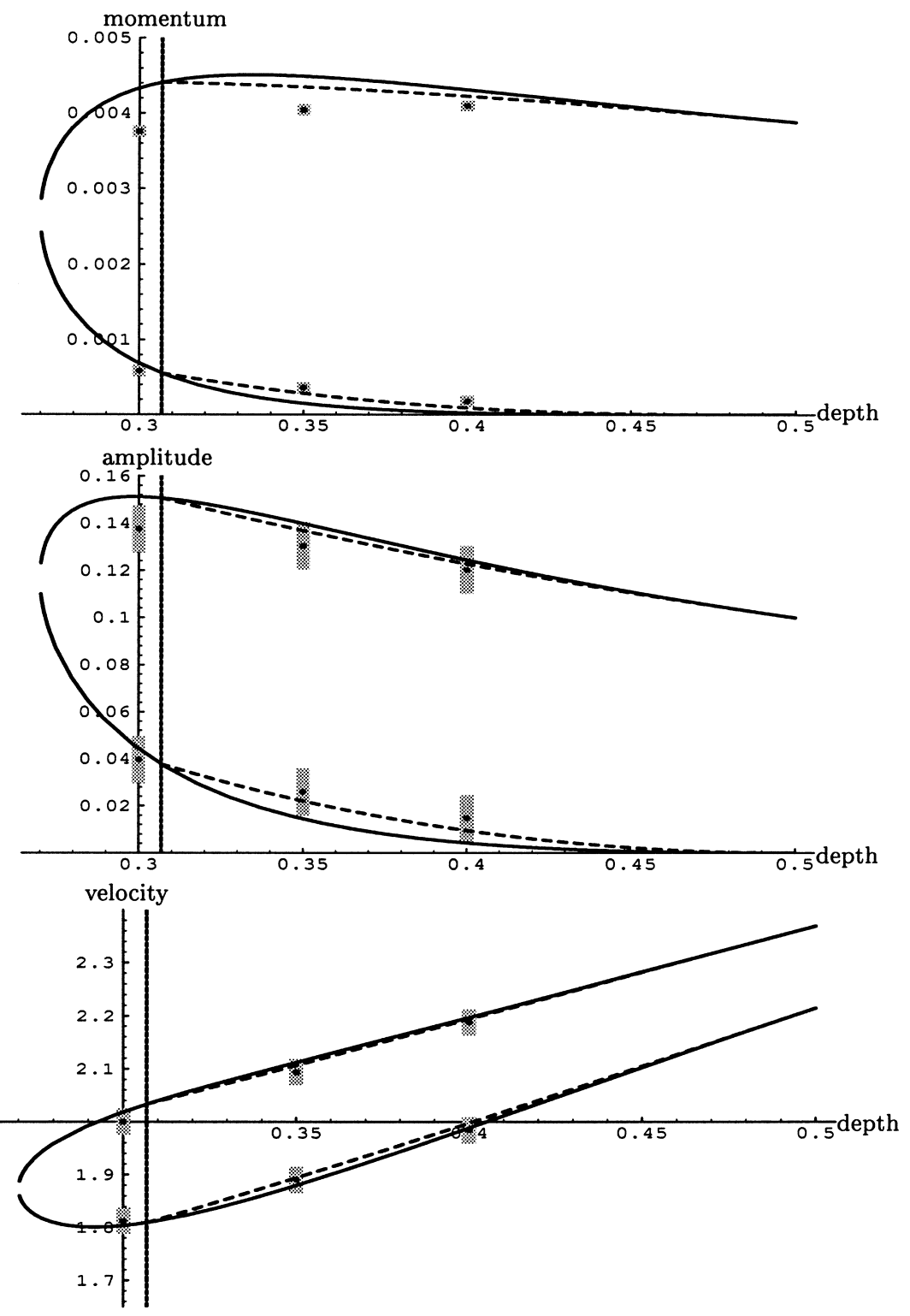

Fig. 7. Two-soliton parameters obtain from the Q-H two-soliton approximation (solid lines), the IS results (dashed lines), and the numerical computations (dots). The error bars around the dots show the possible deviations. The vertical lines $h=\hbar_{2}$ indicate the eigendepth for the pure two-soliton of the KdV model, obtained from IS.

the result of the inverse scattering theory is that the initial soliton will emerge to $N$-soliton with an oscillating tail if $h_{1} \in\left(\hbar_{N}, \hbar_{N-1}\right)$, and it will emerge to exactly $N$-soliton if $h_{1}=\hbar_{N}$. Hence, on an interval $\left[\hbar_{2}, h_{0}\right]$, with $h_{0}=0.5$, we have two-soliton with an oscillating tail, and we can calculate the amplitudes, momenta and velocities of each single-soliton; the results are plotted as dashed-lines in Fig. 7 .

In Fig. 7 the momenta of the two single-solitons given by (21) are plotted as full lines. We also plot the corresponding amplitudes and velocities. The two-soliton data obtained numerically are plotted in dots. Error bars around the dots show the possible deviation caused by a simple method applied in finding the heights and the positions of wave crests. 

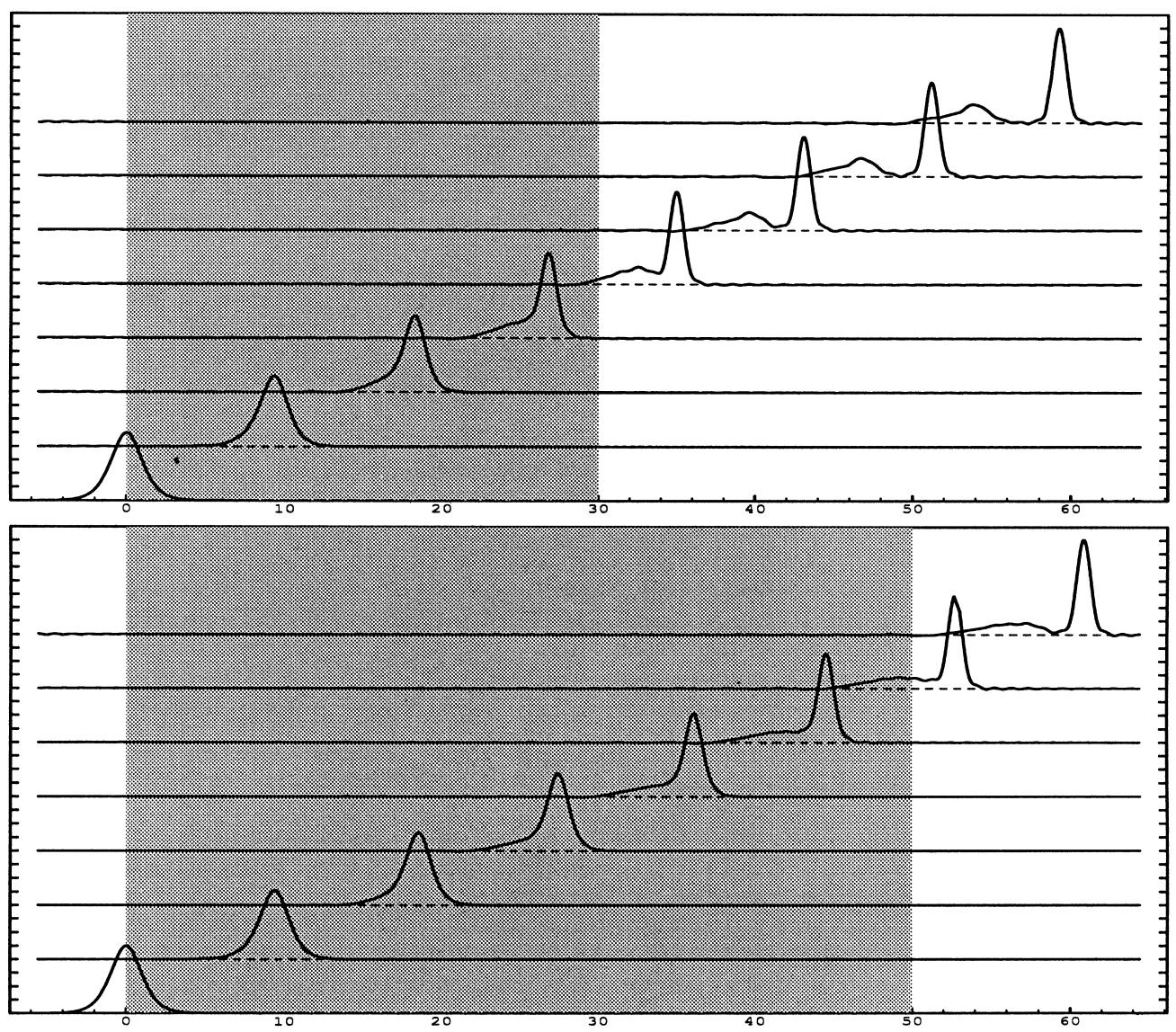

Fig. 8. Solitary wave running up from $h_{0}=0.5 \mathrm{~m}$ to $h_{1}=\hbar_{2}=0.3068 \mathrm{~m}$, with $L=30 \mathrm{~m}$ (top) and $L=50 \mathrm{~m}$ (bottom).

From Fig. 7, apart from some differences in the amplitudes (and hence the momenta) of the larger waves for $h_{1}=0.3 \mathrm{~m}$ and $h_{1}=0.35 \mathrm{~m}$, we can still conclude that quantitatively the analytical (both the Q-H approximation and the IS approach) and numerical results agree with each other quite well. Note that at the eigendepth $\hbar_{2}$ (indicated by the vertical line), the Q-H approach and the IS method yield exactly the same two-soliton parameters. This is because at $\hbar_{2}$ the actual profile is a pure two-soliton (without an oscillating tail), therefore both approaches give the same results. Since in the Q-H two-soliton approximation, the oscillating tail is neglected, we notice from Fig. 7 (top) that the total momentum $\gamma_{+}(c)+\gamma_{-}(c)$ for $h_{1} \in\left(\hbar_{2}, h_{0}\right)$ is a bit larger than the total momentum obtain from the IS approach.

\section{Discussions on the validity of the quasi-homogeneous approximation}

In order to have a better feeling about the dependence of the solitary waves evolution on the properties of the topography, in this paragraph we present several other numerical computations. We compute a solitary wave running up over a very mildly decreasing depth. Let $L$ denote the length of region of decreasing depth. Previous computations use $L=10 \mathrm{~m}$, here we will use $L=30 \mathrm{~m}$ and $L=50 \mathrm{~m}$. Just like the previous computations, the initial profile is taken to be a solitary wave that is constructed from a lifted-cnoidal wave of amplitude $0.1 \mathrm{~m}$. In order to have the pure two-soliton or three-soliton emerge from the initial wave, we choose $h_{1}$ to be the eigendepths $\hbar_{2}$ or $\hbar_{3}$. 
Consider Fig. 8 in which the wave is running up from $h_{0}=0.5 \mathrm{~m}$ to $h_{1}=\hbar_{2}=0.3068 \mathrm{~m}$, with $L=30 \mathrm{~m}$ (top) and $L=50 \mathrm{~m}$ (bottom). We observe the appearance of a significant hump, like a 'flat' tail behind the wave, which is clearly observable in Fig. 8 (bottom). When the wave arrives at the flat region with a depth $h_{1}$, the tail starts to separate form the wave, and it slowly deforms into a single-soliton profile.

Another computation that also shows the appearance of this tail was done using $h_{1}=\hbar_{3}$, the eigendepth for three-soliton, and the results are plotted in Fig. 9. Despite the wiggles around the wave, caused by the technical restriction to 64 Fourier modes, we again observe clearly the 'flat' tail behind the wave. After the wave arrives at the flat region the three-soliton emerge, see the last wave profile of Fig. 9 (top).

Fig. 10 shows the calculations using $h_{1}=\hbar_{2}$ and $h_{1}=\hbar_{3}$ with $L=10 \mathrm{~m}$. The results show clearly that the initial solitary wave emerge into the pure two-soliton and three-soliton, despite the fact of the limited number of Fourier modes that produce some wiggles in the steeper wave, see Fig. 10 (bottom).

In Fig. 8 we show that during a milder run up, the wave profile looks like a solitary wave with a 'flat' tail. Nonetheless, it still make sense to approximate this profile with the two-soliton shape; look at Fig. 11 where we plot the two-soliton shape at a characteristic instant after interaction.

The Q-H two-soliton approximation describes this phenomena as follows. The hump behind the wave is the 'second' solitary wave that starts to grow directly after the solitary wave enters a region of decreasing depth, recall Fig. 5(a). The two parameters determining the wave profile, $\gamma_{+}$and $\gamma_{-}$are changing as functions of $h=h(\varphi)$. So while running up the two-soliton shapes are developing (the two constituent single-solitons remain in an interaction
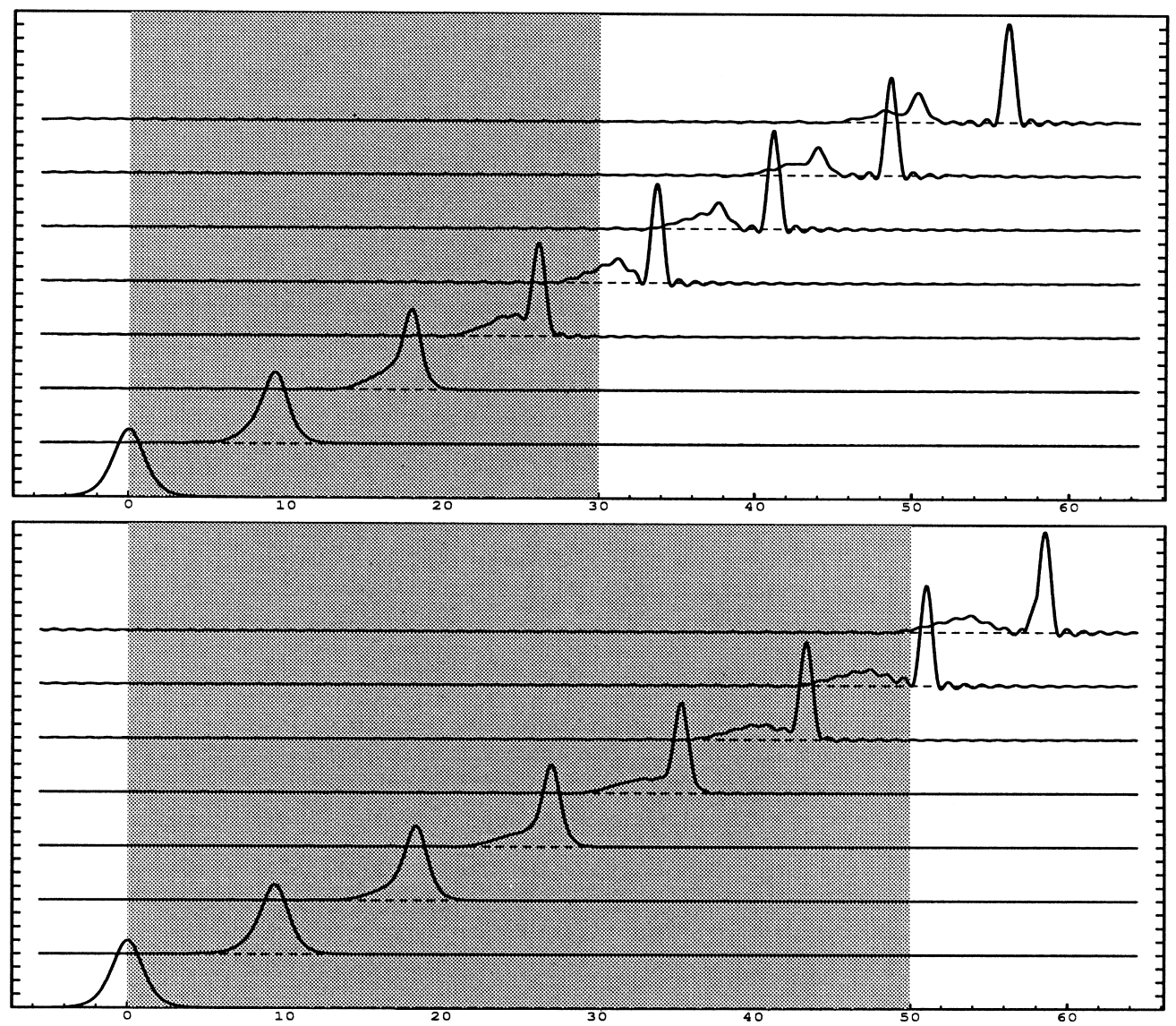

Fig. 9. Solitary wave running up from $h_{0}=0.5 \mathrm{~m}$ to $h_{1}=\hbar_{3}=0.2255 \mathrm{~m}$, with $L=30 \mathrm{~m}$ (top) and $L=50 \mathrm{~m}$ (bottom). 

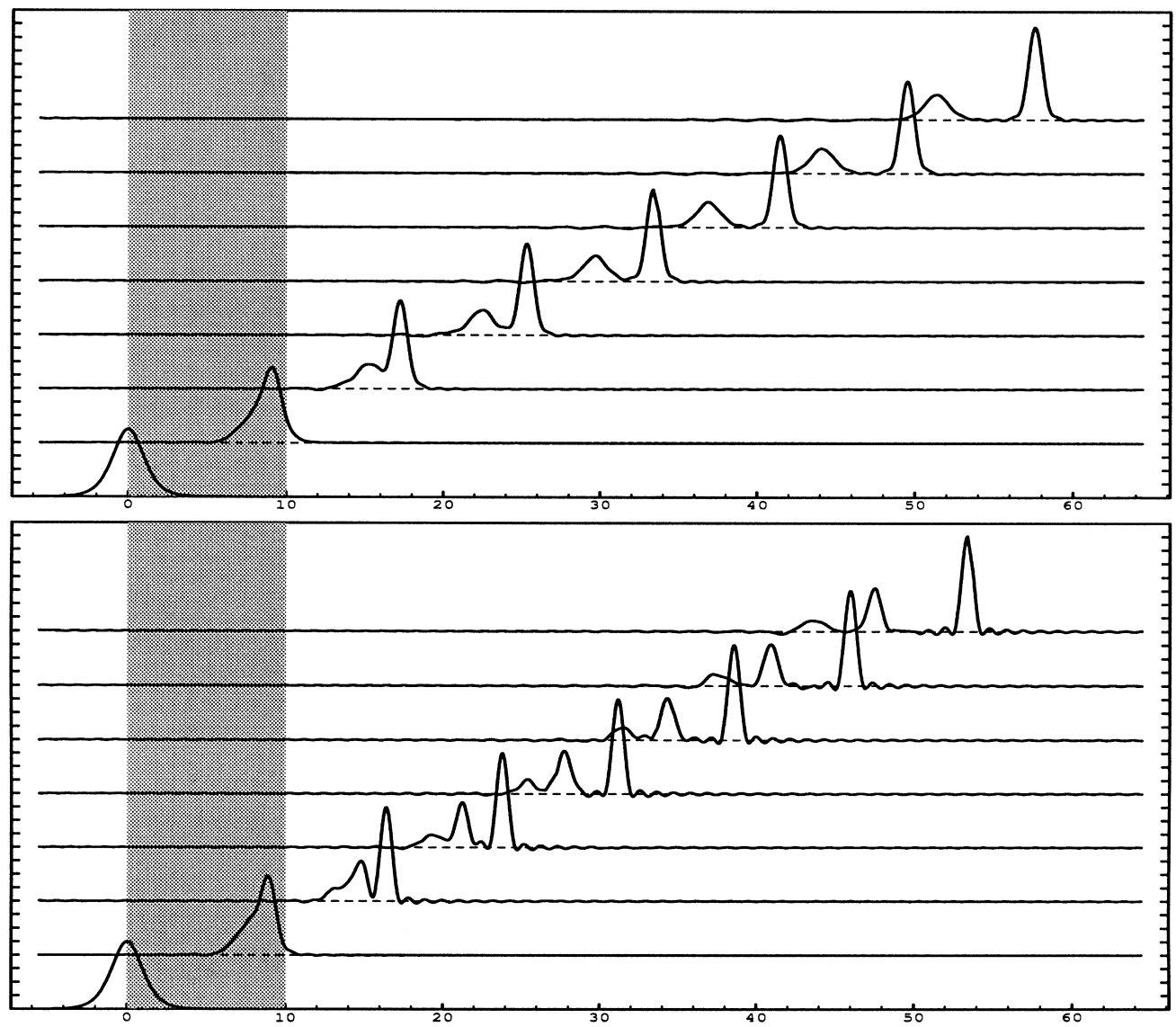

Fig. 10. Solitary wave running up to a flat bottom with a depth $h_{1}=\hbar_{2}=0.3068 \mathrm{~m}$ (top) and depth $h_{1}=\hbar_{3}=0.2255 \mathrm{~m}$ (bottom).

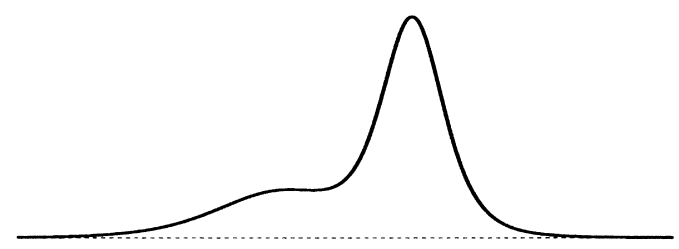

Fig. 11. A two-soliton shape at a time of interaction at which the shape looks like a solitary wave with a 'flat' tail.

process) till the wave enters the region where the bottom is flat again with a depth $h_{1}$. At that time, the wave profile is parameterized by $\gamma_{-}\left(h_{1}\right)$ and $\gamma_{+}\left(h_{1}\right)$, and then the two single-solitons start to separate.

This 'flat' tail will be observable if the bottom variation occurs over a long distance. For a depth change that occurs over a short distance, the tail looks like a small disturbance attached on the left side of the wave, see Fig. 10. In both cases, when the waves arrive at the flat region, two-solitons (or $N$-solitons, with $N>2$, see discussion below) will emerge.

The minimal depth for which the Q-H two-soliton approximation is still valid is determined using the IS results: for $h_{1} \in\left[\hbar_{2}, h_{0}\right)$, the solitary wave will develop into a two-soliton with an oscillating tail. Therefore, in the Q$\mathrm{H}$ approximation for $h_{1} \in\left(\hbar_{2}, h_{0}\right]$, it is reasonable to approximate the wave profile with the pure two-soliton shape. We stress here that in applying the Q-H approximation, we need to choose a priori suitable base functions. 
Based on the IS result, for $h_{1} \in\left[\hbar_{N}, \hbar_{N-1}\right.$ ), with $N \geq 2$, we should apply the Q-H approximation of $N$-soliton shapes.

The dip behind the second soliton in Fig. 3 (top), which is much smaller compared to those in Fig. 3 (middle) and (bottom), can now be explained because the depth $h_{1}=0.3 \mathrm{~m}$ is close to the eigendepth $h_{1}=\hbar_{2}$ compared with $h_{1}=0.35 \mathrm{~m}$ and $h_{1}=0.4 \mathrm{~m}$.

If for $h_{1}<\hbar_{2}$ we still apply the two-soliton approximation, meaning that we distribute the total momentum among the two single-solitons instead of with more solitons, we will make an error. But if $h_{1} \in\left[\hbar_{2}, h_{0}\right)$ and we apply $N$-soliton approximation, with $N \geq 3$, this will be correct, since two-soliton shapes are special cases of $N$-soliton shapes, i.e. the manifold of $N$-soliton shapes is an unfolding of the manifold of two-soliton shapes. Applying a Q-H $N$-soliton approximation, with $N \geq 3$, in principle will be the same with the Q-H two-soliton, but it will involve $(2 N+1)$ parameters and determining the evolution of these parameters will be much more difficult.

When the wave is in the process of running up, one cannot tell which value of $N$ gives the best approximation for describing the whole process. The knowledge of the depth $h_{1}$ leads us to a value for $N$, in the sense that after the wave enters the constant depth $h_{1}$, the wave profile is approximated best with (at least) $N$-soliton profile. Hence, simply using the Q-H $N$-soliton approximation, even during the run up process, seems to be a good choice.

Concerning the applicability of the Q-H two-soliton approximation, observe the following. Note that the computation for $h_{1}=0.3 \mathrm{~m}$ in Section 3 turns out to be in the area of three-soliton with an oscillating tail, since $h_{1}$ is less than, although close to, $\hbar_{2}=0.3068 \mathrm{~m}$. But in the numerical result, Fig. 3 (top), the wave profiles still look very much like two-solitons. Therefore, applying the Q-H based on two-soliton shapes is still reasonable. Moreover, the amplitude of the large wave is approximately $0.15 \mathrm{~m}$, see Fig. 7, which is half of the water depth. When $h_{1}$ is less than $0.3 \mathrm{~m}$, in which the $N$-soliton approximation, with $N \geq 3$, should be applied, the water depth $h_{1}$ will be very shallow compared with the wave amplitude. On the other hand, the KdV-top model is not a model for waves with large amplitudes above very shallow water depths.

\section{References}

[1] E. van Groesen, S.R. Pudjaprasetya, Uni-directional waves over slowly varying bottom. Part I. Derivation of a KdV-type of equation, Wave Motion 18 (1993) 345-370.

[2] S.R. Pudjaprasetya, E. van Groesen, Uni-directional waves over slowly varying bottom. Part II. Quasi-static approximation of distorting waves, Wave Motion 23 (1996) 23-38.

[3] S.R. Pudjapraseta, Evolution of waves above slightly varying bottom: a variational approach Ph.D. Thesis, University of Twente, The Netherlands, 1996.

[4] F.P.H. van Beckum, Hamiltonian-consistent discretisation of wave equations, Ph.D. Thesis, University of Twente, The Netherlands, 1995.

[5] R.S. Johnson, On the development of a solitary wave moving over an uneven bottom, Proc. Cambridge. Phil. Soc. 73 (1973) $183-203$.

[6] F.P.H. van Beckum, W. Djohan, WAVEPACK, software and manual for calculation of wave problems, University of Twente, 1994.

[7] E. van Groesen, A phenomenological description of soliton splitting during run up, Contemp. Math. (1996) 211-222.

[8] R.S. Johnson, Some numerical solutions of a variable-coefficient Korteweg-de Vries equation (with application to solitary wave development on a shelf), J. Fluid Mech. 54 (1972) 81-91. 